\title{
Observations of traveling ionospheric disturbances and 3-m scale irregularities in the nighttime $F$-region ionosphere with the MU radar and a GPS network
}

\author{
A. Saito ${ }^{1,2}$, M. Nishimura ${ }^{3 *}$, M. Yamamoto ${ }^{3}$, S. Fukao ${ }^{3}$, T. Tsugawa ${ }^{4}$, Y. Otsuka ${ }^{5}$, S. Miyazaki ${ }^{6}$, and M. C. Kelley ${ }^{1}$ \\ ${ }^{1} 304$ Rhodes Hall, School of Electrical and Computer Engineering, Cornell University, Ithaca, NY 14853-3801, U.S.A. \\ ${ }^{2}$ On leave from Department of Geophysics, Graduate School of Science, Kyoto University, Kyoto 606-8502, Japan \\ ${ }^{3}$ Radio Science Center for Space and Atmosphere, Kyoto University, Uji 611-0011, Japan \\ ${ }^{4}$ Department of Geophysics, Graduate School of Science, Kyoto University, Kyoto 606-8502, Japan \\ ${ }^{5}$ Solar-Terrestrial Environment Laboratory, Nagoya University, Aichi 442-8507, Japan \\ ${ }^{6}$ Geographical Survey Institute, Ibaraki 305-0811, Japan
}

(Received October 31, 2000; Revised September 20, 2001; Accepted October 1, 2001)

\begin{abstract}
The nighttime traveling ionospheric disturbances (TIDs) and the $F$-region 3-m scale field-aligned irregularities were simultaneously observed with the MU radar and GEONET, a GPS network, during the FRONT (F-region Radio and Optical measurement of the Nighttime TID) campaign periods in May 1998 and August 1999. The vertical profile of electron density detected by the incoherent scatter observation of the MU radar clarified that ionized atmosphere on the bottomside of the ionospheric $F$-region was deeply modulated by TIDs, which would cause the variations of the $630 \mathrm{~nm}$ band airglow luminosity. The coherent echoes from the 3-m scale fieldaligned irregularities were detected also on the bottomside of the $F$-region in the nights when TIDs were intense in amplitude and the ionosphere was uplifted. Two-dimensional structures of the field-aligned irregularities detected by the multi-beam observation of the MU radar revealed that the 3-m scale irregularities formed band-like structures and traveled to the southwest in several nights. Their wave vector and traveling velocity were coincident with those of the nighttime TIDs that were simultaneously detected by the TEC observation of GEONET. The intense Doppler velocities of the coherent echoes indicate that the polarization electric field is generated inside the TIDs. We consider that the horizontal gradient of the electric conductivity associated by TIDs and the vertical gradient of the conductivity on the bottomside of the $F$-region ionosphere generates the 3-m scale irregularities through the gradient-drift instability process. The anti-correlation of the occurrence rate of the $F$-region field-aligned irregularities to the solar activity would be caused by the anti-correlation of the amplitude of TIDs and of the vertical gradient of the Pedersen conductivity.
\end{abstract}

\section{Introduction}

Nighttime traveling ionospheric disturbances (TIDs) at mid-latitudes have been observed in several longitudinal sectors and with several observational techniques in recent years (Jacobson et al., 1995; Miller et al., 1997; Saito et al., 1998; Garcia et al., 2000). Interest has grown because their features cannot be explained by the classical theory proposed by Hines (1964) (Kelley and Miller, 1997; Saito et al., 2001). GEONET (GPS Earth Observation Network), a dense and wide-area GPS receiver network in Japan, has provided high-resolution two-dimensional total electron content (TEC) maps with 30 seconds time resolution since 1997 (Saito et al., 1998). The two-dimensional TEC maps have revealed that in the nighttime the clear wave fronts of TEC propagate to the southwest with velocities of about $100 \mathrm{~m} / \mathrm{s}$ and have wavelengths of a few hundred kilometers. Considering these scale and propagation characteristics, this phenomenon is categorized to the

*Now at NTT Communicationware Co., Japan.

Copy right $($ C) The Society of Geomagnetism and Earth, Planetary and Space Sciences (SGEPSS); The Seismological Society of Japan; The Volcanological Society of Japan; The Geodetic Society of Japan; The Japanese Society for Planetary Sciences. medium scale traveling ionospheric disturbances (MSTIDs) (Francis, 1974). All-sky imagers have detected similar traveling structures in $630 \mathrm{~nm}$ band airglow at midlatitudes (Mendillo et al., 1997; Taylor et al., 1998; Garcia et al., 2000). The wavelength and propagation velocity are similar to those of the TEC structures (Garcia et al., 2000). The $F$-region 3-m scale field-aligned irregularities (FAIs) are another traveling disturbance in the nighttime mid-latitude ionosphere, which were detected by the MU radar (Fukao et al., 1988; Fukao et al., 1991). Mid-latitude $F$-region FAIs were detected also in the Caribbean sector with two VHF radars (Swartz et al., 2000).

These three phenomena measured by independent observational techniques occur at same location and same local time, and have similar propagation characteristics. The FRONT (F-region Radio and Optical measurement of the Nighttime TID) campaign was conducted to clarify the relationship between these three phenomena with multiinstruments at multi-locations (Kubota et al., 2000; Saito et al., 2001). Airglow structures were measured with five or six all-sky CCD cameras over Japan during the FRONT campaign periods in May 1998 and in August 1999, respectively. The MU radar observed the vertical profile of elec- 


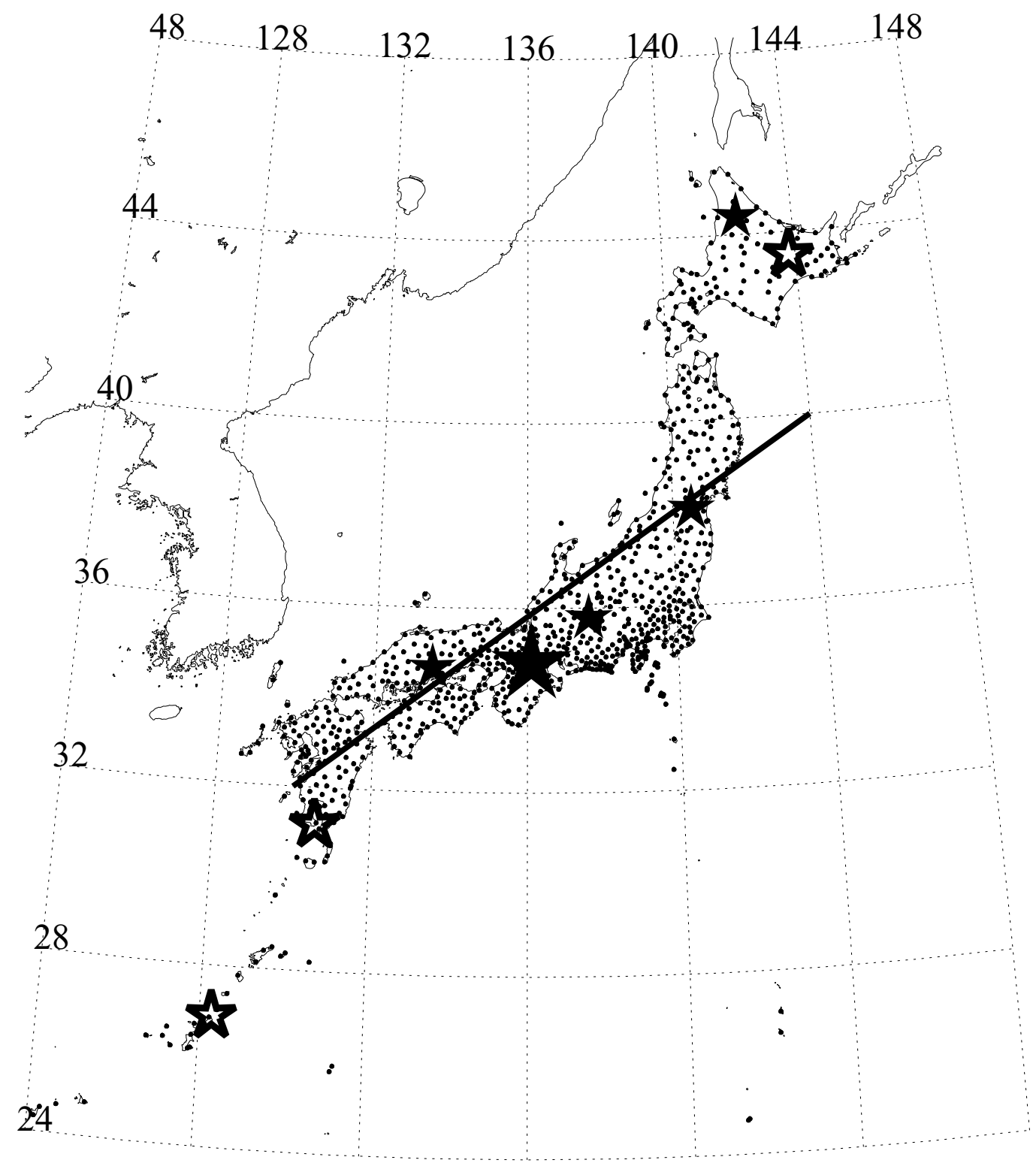

Fig. 1. Observational sites of the FRONT campaign. The locations of the GPS receivers of GEONET are represented by small solid circles. The MU radar site is represented by a large solid star mark. Solid star marks indicate the locations of the optical measurement sites for the FRONT-1 campaign and open star marks indicate additional sites for the FRONT-2 campaign. TEC along the solid line over Japan on May 18/19, 1998 is displayed in Fig. 6.

tron density in its incoherent scatter mode and the 3-m scale $F$-region FAIs in its coherent scatter mode. GEONET observed the two-dimensional distribution of the TEC. The preliminary results of the FRONT campaign showed good spatial coincidence between TEC structures and the $630 \mathrm{~nm}$ band airglow structures (Saito et al., 2001). This coincidence indicates that the airglow variations are caused by plasma density variations in the emitting layer of the OI band, centered $260 \mathrm{~km}$ altitude (Kubota et al., 2000). Ogawa et al. (2002) quantitatively compared the variations of 630 $\mathrm{nm}$ band airglow emission and TEC observed with GPS using the SUPIM model. Shiokawa et al. (2002) studied the relation between $630 \mathrm{~nm}$ airglow and TEC at the northern edge of the equatorial anomaly. Saito et al. (2001) reported that the TEC structures evolved as they traveled to the southwest over Japan. This indicates the existence of a mechanism that keeps the structures growing. The correlation between the occurrence of the $F$-region FAIs and the amplitude of TEC structures was also reported by Saito et al. (2001). The $F$-region FAIs were detected in the time periods when the amplitude of TEC variations were largest in the nine night FRONT-1 campaign.

Following these preliminary results, this paper intends to clarify the relationship between the TIDs, $F$-region FAIs and the structure of the background ionospheric electron density, using the observations of the coherent and incoherent scatter echoes by the MU radar, and of TEC by GEONET.

\section{Observational Setup}

The FRONT campaigns were conducted in the period from May 16 to 24, 1998 (FRONT-1) and from August 8 to 18,1999 (FRONT-2). The locations of the observational sites are displayed in Fig. 1. Star marks show the site lo- 
cations of the optical measurements. Five solid star marks represent the sites in the FRONT-1 period. Open star marks are additional sites for the FRONT-2. The large solid star mark represents the location of the MU radar, Shigaraki (Lat: $34.8^{\circ}$, Long: $136.1^{\circ}$, M-Lat: $25.6^{\circ}$ ). The detailed descriptions on these optical sites and their results in the FRONT campaign were presented by Kubota et al. (2000), Saito et al. (2001), Ogawa et al. (2002), and Shiokawa et al. (2000, 2002). Small solid circles represent the locations of the GPS receivers of GEONET. The sampling period of these GPS receivers was 30 seconds. To exclude the errors caused by the cycle slips and the accuracy of the mapping function that is used to convert the observed line-of-sight TEC to the vertical TEC only the data whose elevation angle is larger than 45 degrees are used in this analysis. The average separation of the receivers is about $25 \mathrm{~km}$. The MU radar at Shigaraki was operated in a mode, which consisted of 30 seconds of the coherent scatter observation and 150 seconds of the incoherent scatter observation with $50 \mathrm{sec}-$ onds of interruptions for switching between the two observations. In the incoherent observation, the radar beam points the zenith direction. In the coherent observation, the radar beam was steered to the north with about 60 degrees of the zenith angle to cross the geomagnetic field line perpendicularly. Since the MU radar is a phased-array radar, it can steer the radar beam virtually simultaneously in several directions. In the coherent scatter observations of the FRONT campaign, sixteen beams were utilized to detect the twodimensional distribution of the $F$-region FAIs in the region between $36^{\circ}$ and $40^{\circ}$ in latitude, and $132^{\circ}$ and $139^{\circ}$ in longitude. The fifteen of sixteen radar beams were made perpendicular to the geomagnetic field line at $275 \mathrm{~km}$ altitude, and had azimuthal angles between \pm 25 degrees around the geomagnetic north. The remaining one beam had a larger zenith angle than that of others to cross the geomagnetic field line perpendicularly at $375 \mathrm{~km}$ altitude and to detect altitudinal extension of the $F$-region FAIs.

\section{Observational Results}

\subsection{Vertical structure of the traveling ionospheric dis- turbances}

Two-dimensional maps of the perturbation component of TEC derived with GEONET and the GPS satellite PRN22 at 2340 JST on August 8, 1999 and that 25 minutes later are shown in Figs. 2(a) and (b), respectively. The largescale TEC variations whose time scale was longer than one hour were subtracted to derive the perturbation component of TEC. The derivation method of these two-dimensional TEC perturbation maps was described in detail in Saito et al., (1998). The TEC data of this paper are averaged with its nearby eight grids to display the alignment of the wave fronts clearly. This does not significantly affect the result since the typical scale size of TEC structures on which we focus in this paper are sufficiently larger than the grid size, $0.15^{\circ} \times 0.15^{\circ}$ in latitude and longitude. As many as five wave fronts of TEC, three weak enhancements in the western part of Japan, an intense one at the middle and another front in the northern part, can be seen in Fig. 2(a). They were stretching from the northwest to the southeast and their wavelengths were from $200 \mathrm{~km}$ to $500 \mathrm{~km}$. They traveled to the southwest direction in about $100 \mathrm{~m} / \mathrm{s}$. These characteristics of the wave fronts of TEC are similar to those of MSTIDs that have been frequently detected by GEONET since 1997 (Saito et al., 1998, 2001). The peak-to-peak amplitude of these TIDs was about one TEC unit $\left(10^{16}\right.$ electron $/ \mathrm{m}^{2}$ ). The background value of TEC in this time period was about 21 TEC units, which was estimated with the method developed by Otsuka et al. (2002). The ratio of the perturbation to the background is about $2.5 \%$. Twenty-five minutes corresponds to half of the wave period observed at a fixed location. The MU radar located at Shigaraki represented by the red star mark in Figs. 2(a) and (b), was under a minimum at $2340 \mathrm{JST}$ and a maximum at $0005 \mathrm{JST}$.

The incoherent scatter observation of the MU radar provided the electron density profile over Shigaraki. The altitude-time-intensity plot of the incoherent scatter observation is displayed in Fig. 3 for the time period between 2200 on August 8 and 0200 on the subsequent day, 1999 JST. The incoherent echo intensity is converted to the electron density using the ionosonde data at Kokubunji (Lat: $35.7^{\circ}$, Long: $139.5^{\circ}$ ), which is operated by the Communication Research Laboratory. The $F$-region peak height, which is represented by blue line, was higher than $370 \mathrm{~km}$ during this time period and the electron density was lower than $1 \times 10^{6} / \mathrm{cm}^{3}$. Therefore, the incoherent scatter echoes were so weak that they suffered large statistical fluctuations. To reduce these fluctuations, the data are averaged with a moving window of $23 \mathrm{~km}$ altitude and 45 minutes for this plot. The $F$-region ionosphere was uplifted higher than $380 \mathrm{~km}$ altitude during this time period. In the pre-midnight period, the $F$-region ionosphere ascended and reached $405 \mathrm{~km}$ altitude at 2330 JST while the electron density constantly decreased. After $2330 \mathrm{JST}$, the ionosphere gradually descended and the electron density showed temporal fluctuations. The red line indicates the perturbation component of TEC derived with GEONET and the GPS satellite PRN22 in the region between $35.10^{\circ}$ and $35.70^{\circ}$ in latitude, and between $135.85^{\circ}$ and $136.45^{\circ}$ in longitude. This region is north of the MU radar site. The PRN22 satellite flew north of Japan during this time period. There were no TEC data just over the MU radar site as seen in Figs. 2(a) and (b). Therefore, the TEC values at this region are plotted to be compared to the electron density measured over the MU radar. The amplitude of TEC fluctuations was small before 2330 JST. After the ionosphere reached its maximum height in this night at 2330 JST, the TEC showed intense fluctuations. These fluctuations have good agreement to the fluctuations of the electron density observed by the MU radar, though the structures detected by the MU radar data are blurred because the temporal average window size, 45 minutes, is comparable to the temporal scale of the fluctuations. There is no conspicuous vertical phase propagation in these fluctuations of the MU radar data.

To compare the electron density data detected by the MU radar with the TEC data of GEONET with higher temporal resolution, the integrated value of the electron density on the bottomside of the $F$-region ionosphere is plotted along with the TEC data of GEONET between 2300 and 0030 JST in Fig. 4 for a location just north of the MU radar. The solid line represents the height-integrated electron density 


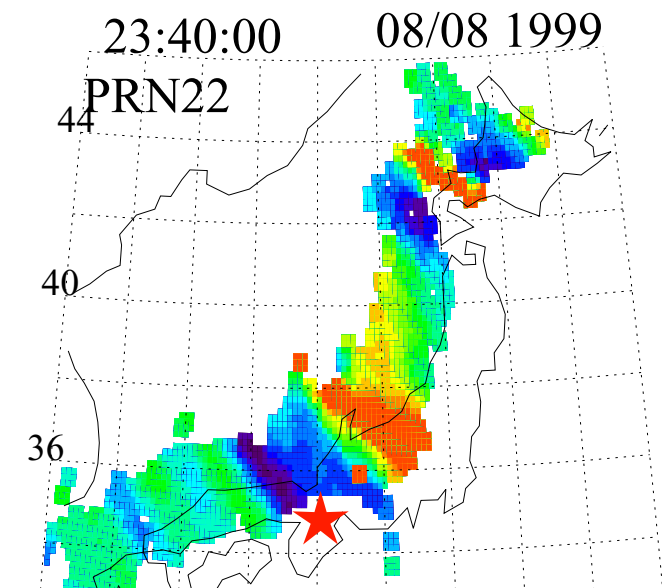

32

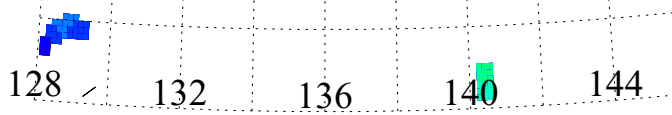

(a)

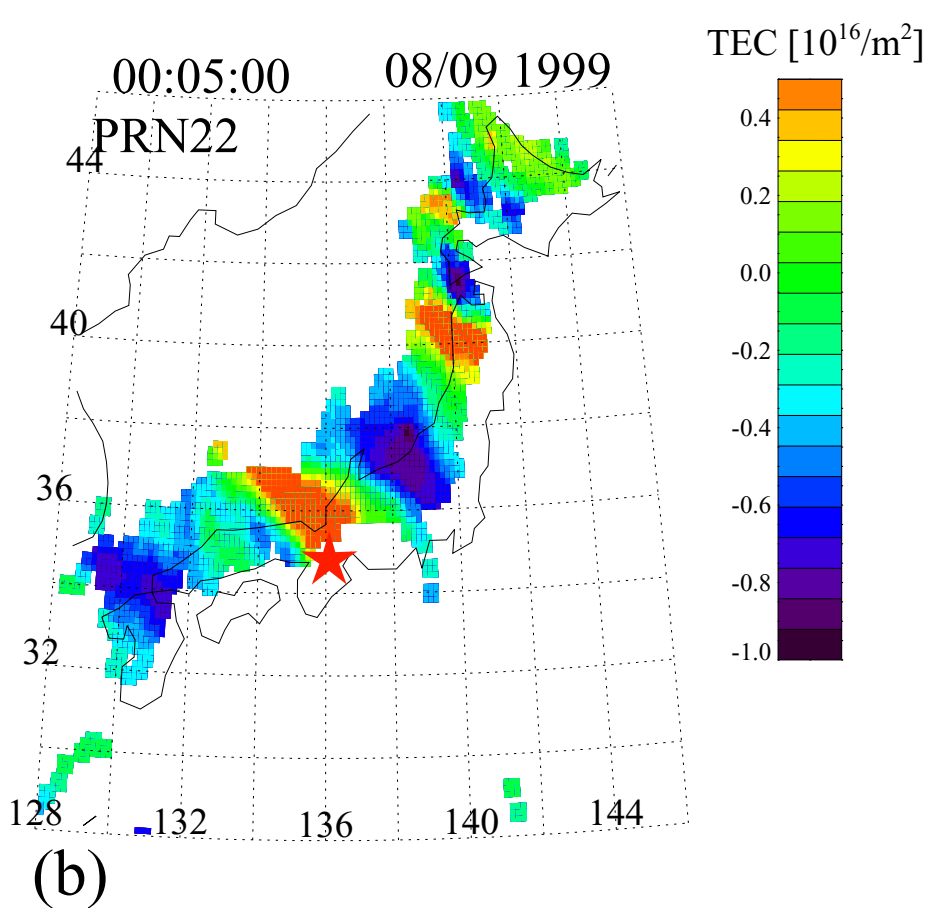

Fig. 2. Two-dimensional distribution of the perturbation component of total electron content detected with GEONET at (a) 23:40:00 on August 8, 1999 and (b) at 00:05:00 on August 9 (b), 1999 [JST].

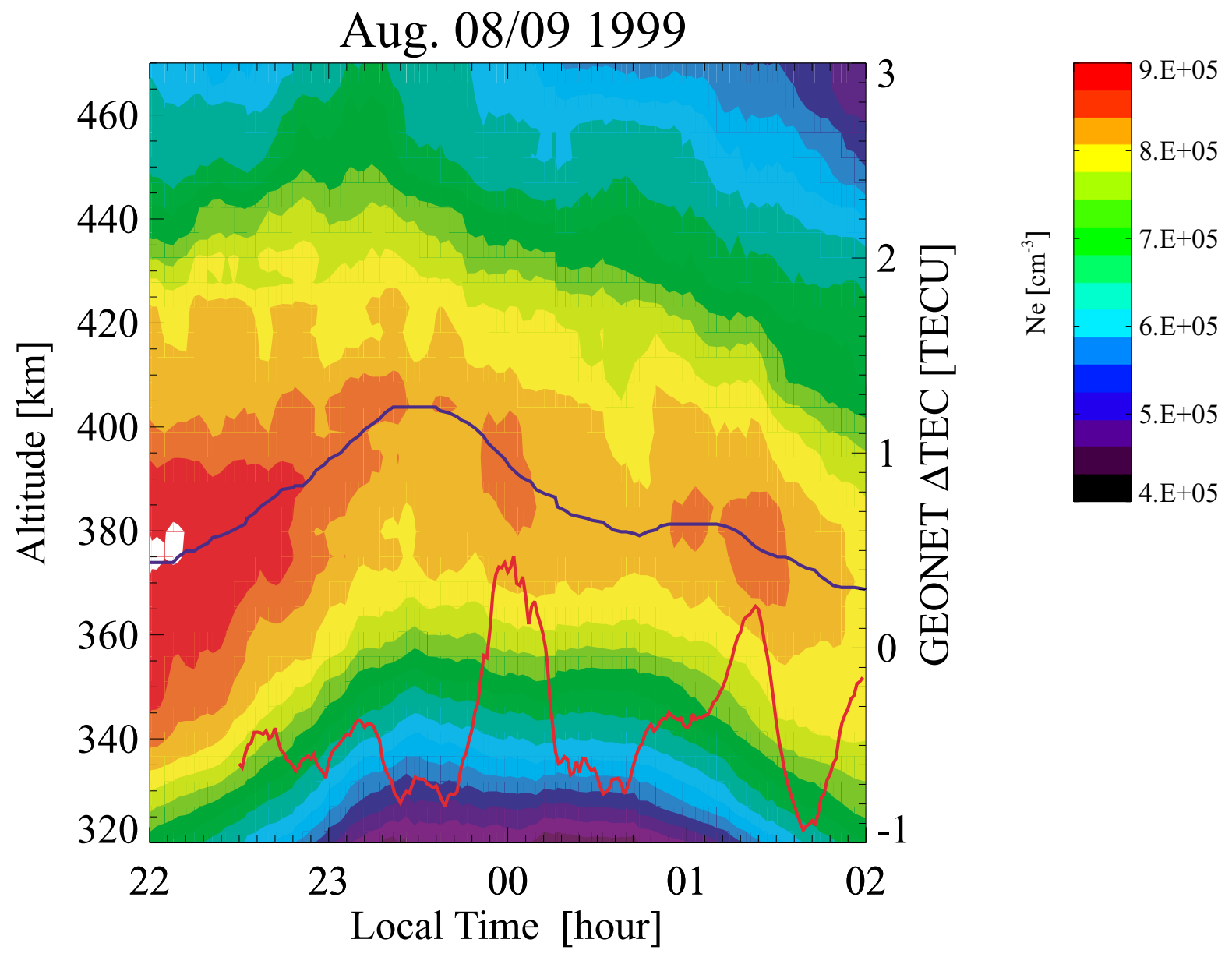

Fig. 3. Contour of the ionospheric electron density detected by the incoherent scatter observation of the MU radar between 2200 on August 8 and 0200 on August 9, 1999 JST. Blue line shows the $F$-region peak altitude. The perturbation component of total electron content nearby the MU radar site detected with GEONET is represented by red line and its scale is displayed on the right-hand side of the plot. 


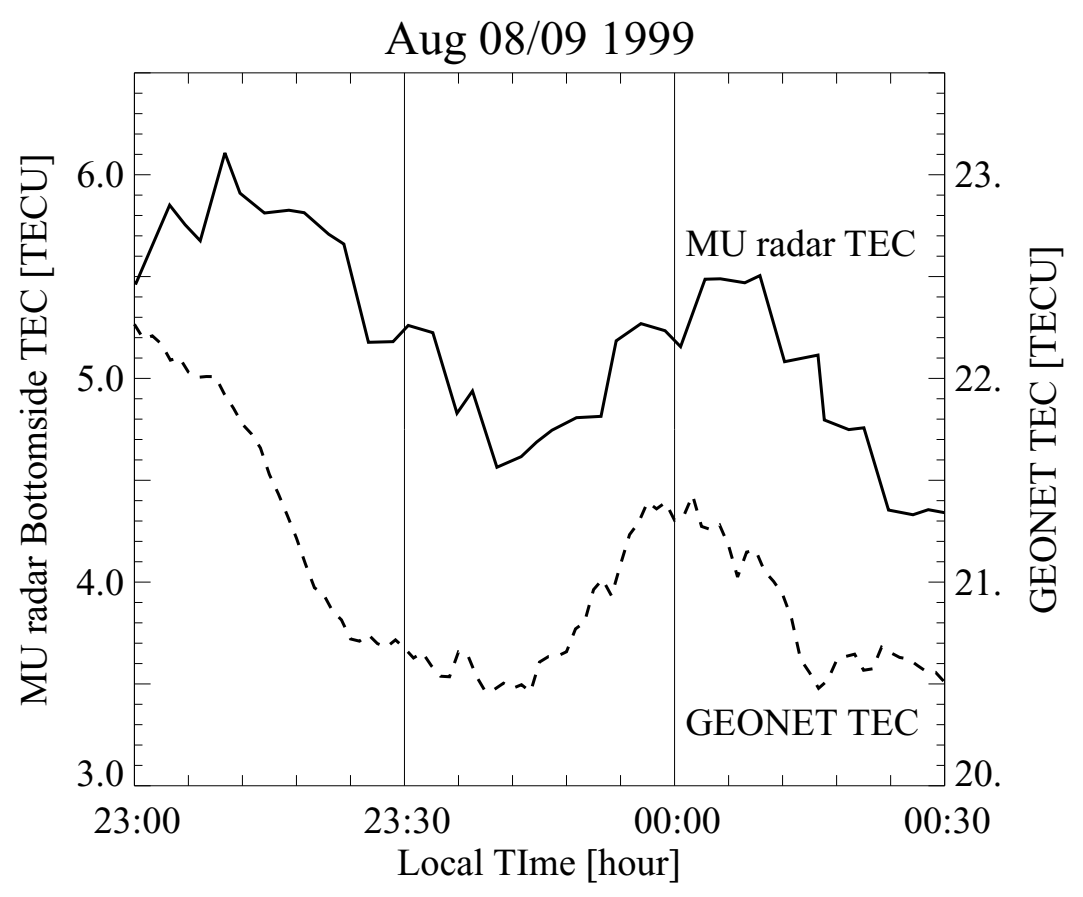

Fig. 4. Height-integrated electron density between $327 \mathrm{~km}$ altitude and the $F$-region peak height derived from the MU radar incoherent scatter observation (solid line) and the absolute value of total electron content in a region nearby the MU radar site (dashed line) between 2300 on August 08 and 0030 on August 09, 1999 JST. The scale for the MU radar TEC is on the left-hand side and the that for the GPS TEC is on the right-hand side.

between the peak altitude of the $F$-region and $327 \mathrm{~km}$ altitude detected by the MU radar and the scale is on the lefthand side of the figure. The dashed line represents the absolute value of TEC in the region just north of the MU radar site and the scale is on the right-hand side. By data integration for the wide altitude range, we can reduce the temporal average window of the MU radar data to 16 minutes with similar data quality. The decrease of the height-integrated electron density before 2340 JST corresponded to the largescale decrease of the electron density whose temporal scale size is longer than one hour, which is seen in the contour of the electron density in Fig. 3 and not seen in the perturbation component of TID in Fig. 3. This decrease is caused by the lack of ionization by the solar radiance and the loss of ionized atmosphere by recombination, which is larger at the lower altitudes. The minimum and maximum after 2340 JST correspond to the TID structures shown in Figs. 2(a) and (b). The peak-to-peak amplitude of the total electron content of the bottomside of the ionosphere was about 1 TEC unit and comparable to the TEC variations measured by GEONET. The variations of TEC detected by the GPS network is dominated by the electron density variations around the $F$-region peak and the bottomside of the $F$-region. Although the signal-to-noise ratio of the data in the topside ionosphere was so weak that it was difficult to precisely estimate the amplitude of the variations on the topside of the ionosphere, the electron density variations in topside ionosphere are not so intense as that on the bottomside. The ratio of the amplitude of the electron density variation on the bottomside of the $F$-region ionosphere to the background value was about $10 \%$ in this case, while those of the TEC variations detected by the GPS network was about $2.5 \%$.

\section{2 $F$-region field-aligned irregularities and the back- ground electron density variations}

$F$-region FAIs appeared on four of the nine nights of the FRONT-1 campaign. The altitude-time-intensity plot of the coherent echoes detected by the MU radar in the night of May 18, 1998 is plotted along with the $F$-region peak altitude derived from the incoherent scatter observation in Fig. 5. The coherent echoes from the $F$-region FAIs were detected between 2330 and 0230 JST between $250 \mathrm{~km}$ and $350 \mathrm{~km}$ altitude. The solid line represents the peak altitude of the $F$-region derived in every 30 minutes. The radar beam of the incoherent scatter observation detected the electron density profile in the zenith direction. In the coherent scatter observation, the radar beam pointed northward at about 60 degrees zenith angle to cross the geomagnetic field line perpendicularly, which has a 48.8 degree inclination. Therefore, the horizontal distance between the regions of these two observations was about $500 \mathrm{~km}$ at $300 \mathrm{~km}$ altitude. The peak of the $F$-region was around $330 \mathrm{~km}$ altitude in the early night and started to ascend at 2230 and reached at $420 \mathrm{~km}$, the highest altitude during this night at 0015. After 0015, the $F$-region ionospheric peak gradually descended to 260 $\mathrm{km}$ altitude at 0515 . The time scale of the ionospheric motion was long enough to be represented by measurements every 30 minutes. The similar vertical movement of the ionosphere were detected by the ionosonde at Kokubunji, which is $300 \mathrm{~km}$ east of the MU radar site, between 2300 and 0330 . The scale sizes of the region of the 3-m scale irregularities and MSTIDs are more than $500 \mathrm{~km}$ along the northwest-southeast direction as shown in Figs. 2, 9, and 10. Thus we believe that the difference of the background ionospheric conditions at the observation regions of the incoherent mode observation and the coherent mode observation 


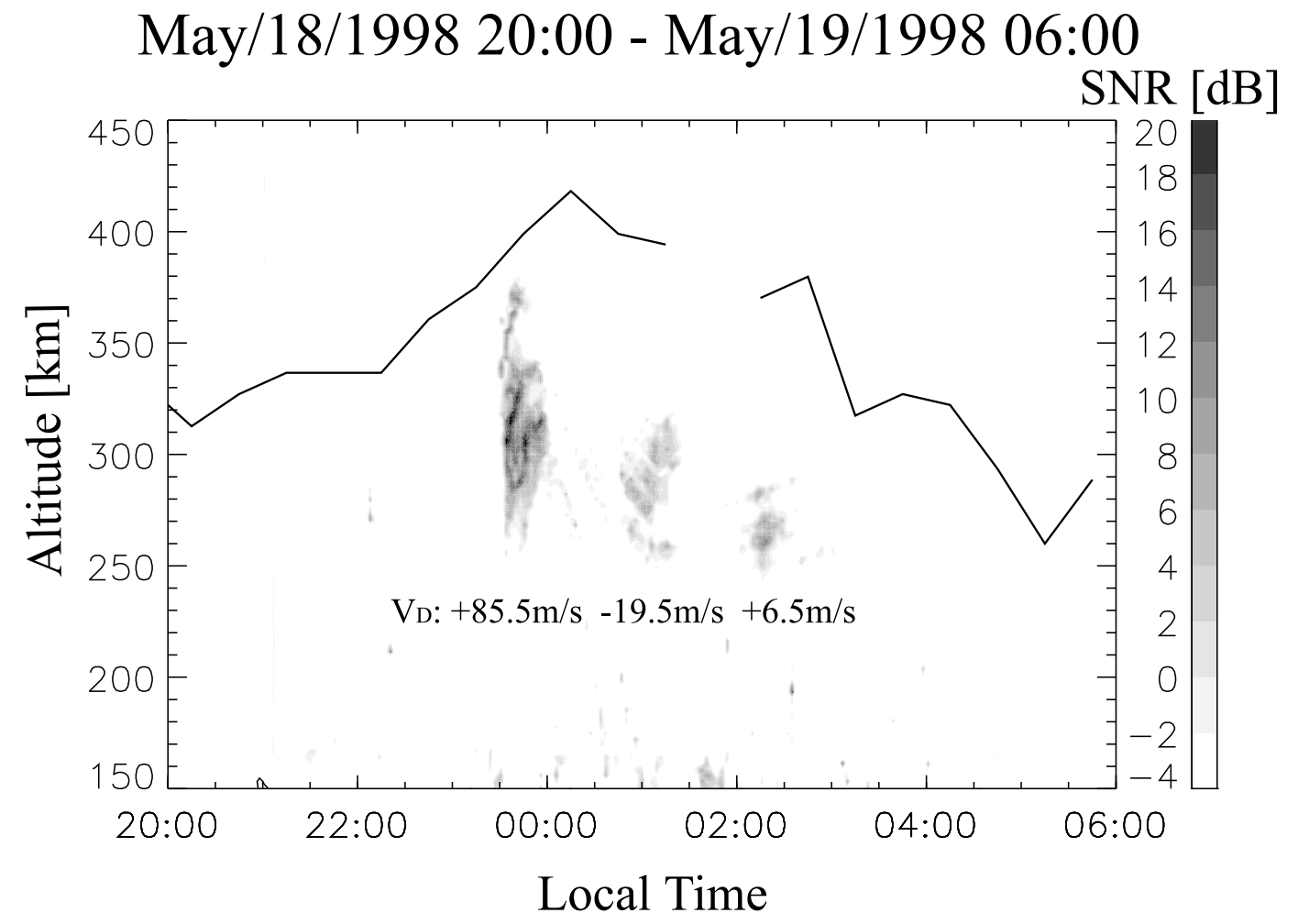

Fig. 5. Altitude-time-intensity plot of the $F$-region field-aligned irregularities detected between 2000 on May 18 and 0600 on May 19 , 1998 JST by the coherent scatter observation of the MU radar. Solid line represents the peak altitude derived from the incoherent scatter observation of the MU radar in every 30 minutes. Average values of the Doppler velocities of coherent echoes for time periods between 2300 and 0000,0030 and 0130 , and 0200 and 0300 are shown below the coherent echoes. The positive Doppler velocities indicate upward/awayward velocities.

was small. The $F$-region FAIs appeared in the time period between 2330 and 0330 when the $F$-region ionosphere was uplifted. It is noted that no FAIs were detected above the $F$-region peak height. The radar beam of the coherent scatter mode crosses the geomagnetic field line around 275 $\mathrm{km}$ altitude. Because of the beam width of the MU radar, the beam has high sensitivity for the altitude range between $195 \mathrm{~km}$ and $345 \mathrm{~km}$ altitude. The coherent echo observation of this beam has low sensitivity at altitudes higher than $345 \mathrm{~km}$. There was, however, another beam with the larger zenith angle of 60.6 degrees to detect the coherent echoes in the higher altitude range between $315 \mathrm{~km}$ and $425 \mathrm{~km}$. The data from this beam confirmed the absence of the coherent echoes at altitudes higher than $380 \mathrm{~km}$.

The average values of the coherent echo Doppler velocities for three time periods, between 2300 and 0000 , between 0030 and 0130 , and between 0200 and 0300 , were $85.5 \mathrm{~m} / \mathrm{s}$, $-19.5 \mathrm{~m} / \mathrm{s}$, and $6.5 \mathrm{~m} / \mathrm{s}$, respectively. In the MU radar coherent echo observations, the Doppler velocities were measured for the upward/awayward velocity to be positive. In the first time period, the maximum of the Doppler velocities was $210 \mathrm{~m} / \mathrm{s}$. This indicates that the intense eastward electric field existed inside this region. The direction of the vertical component of the Doppler velocities were consistent with the vertical motion of the $F$-region uplift measured by the $F$-region peak altitude.

Traveling ionospheric disturbances were detected by the TEC observation by GEONET in this night. Figure 6(a) shows the perturbation component of TEC along a line over
Japan, which is represented by the solid line in Fig. 1. The horizontal axis is the distance along the line and the left end corresponds to the southwestern part of Japan, $32^{\circ}$ in latitude and $129^{\circ}$ in longitude. The right end is at the northeastern part of Japan, $40^{\circ}$ in latitude and $144^{\circ}$ in longitude. The point at $700 \mathrm{~km}$ from the left end corresponds to the region north of the MU radar site. The vertical axis shows the time from 2300 on May 18, 1998 JST at the bottom to 0400 on the subsequent day at the top. The TEC data used in this figure were derived with four GPS satellites, PRN08, PRN09, PRN26, and PRN30. The altitude of the subionospheric point was assumed to be $300 \mathrm{~km}$. Several stripes indicate that the structures of TEC traveled along this line from the northeast to the southwest. The amplitude of these TIDs was high in the time period between 2300 and 0200 . This period was coincident with the period when the $F$-region FAIs were detected and the $F$-region was uplifted.

The incoherent scatter echoes on the bottomside of the $F$-region on this night were significantly contaminated by the coherent echoes of the radar sidelobes from the $3-\mathrm{m}$ scale field-aligned irregularities in the $E$-region ionosphere. The $E$-region FAIs are believed to be generated in association with sporadic $E$ layers and cause intense coherent backscatter echoes in the mid-latitude ionosphere (Fukao et al., 1998). The coherent echoes were so intense that the echoes from sidelobes with large azimuthal angle contaminated the incoherent scatter echoes. Therefore, it is difficult to derive the electron density profile on the bottomside ionosphere and identify the altitudinal range where the 


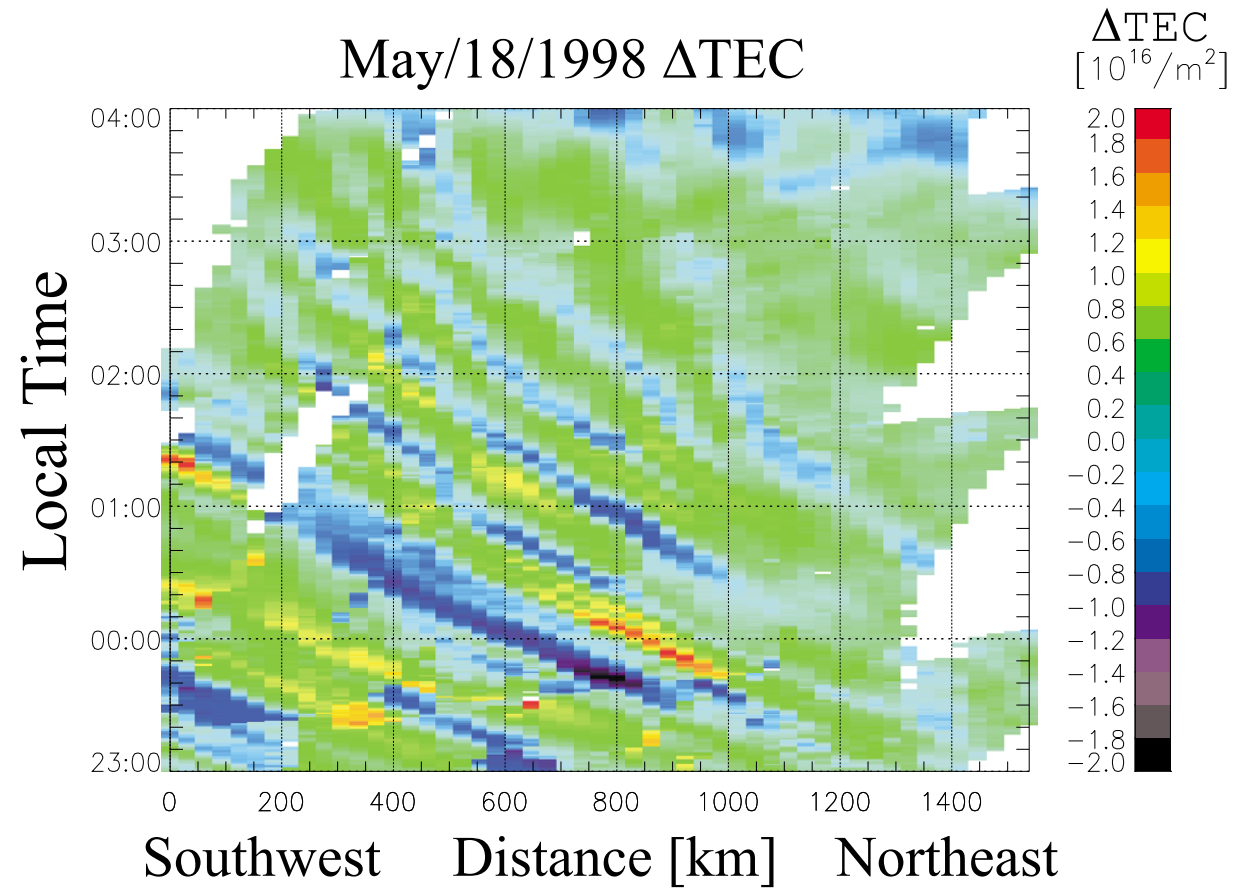

(a)

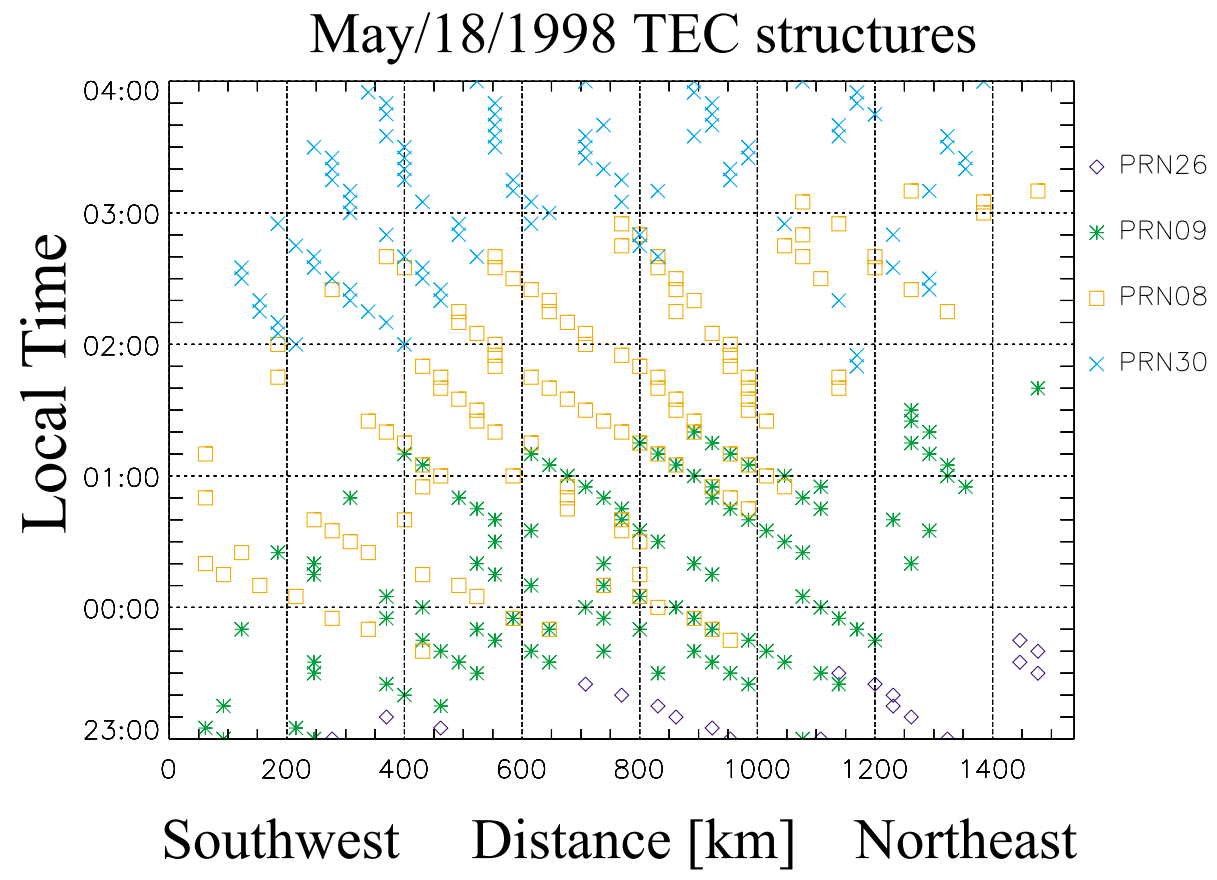

(b)

Fig. 6. (a) Temporal variation of TEC along the line indicated by the solid line in Fig. 1 between 2300 on May 18 and 0400 on May 19 JST, 1998. Right-hand side and left-hand side correspond to the southwest end and the northeast end of the line, respectively. (b) The location of maxima and minima of TEC variations shown in (a). The locations derived with the GPS satellites, PRN26, PRN09, PRN08 and PRN30 are represented by blue diamonds, green asterisks, orange rectangles and blue crosses, respectively. The center altitude of the electron density perturbations is assumed $300 \mathrm{~km}$.

electron density variations of TIDs took place. Instead of the incoherent scatter observation, TEC data from multiplesatellites can provide the information on the altitude region where the electron density variations occurred. The loca- tions of the maxima and minima of the TEC variations are plotted in Fig. 6(b). The maximum and minimum locations derived with the GPS satellites, PRN26, PRN09, PRN08, and PRN30 are represented by blue diamonds, green aster- 

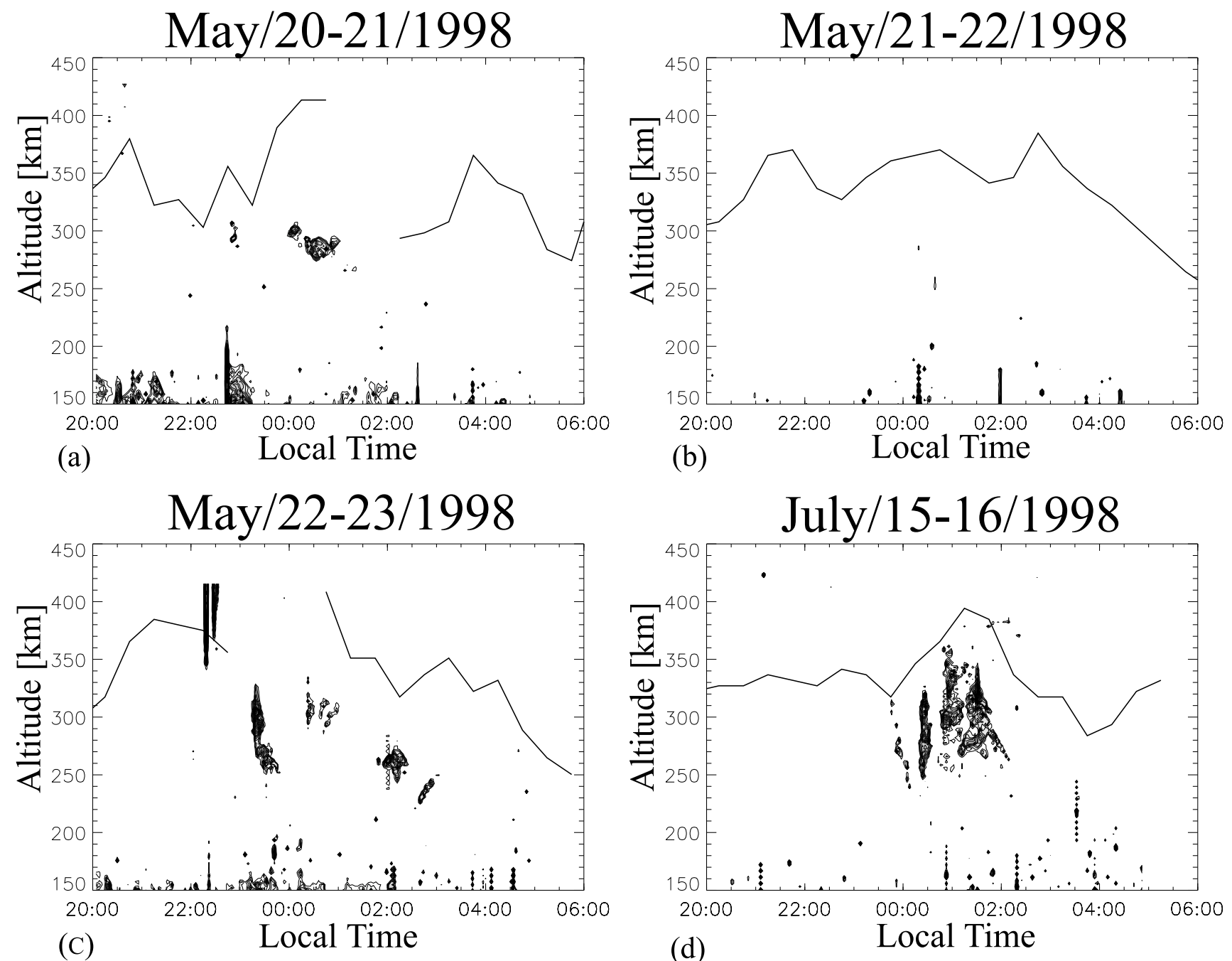

Fig. 7. Same as Fig. 5. for time periods (a) between 2000 on May 20 and 0600 on May 21, 1998 JST, (b) between 2000 on May 21 and 0600 on May 22, 1998 JST, (c) between 2000 on May 22 and 0600 on May 23, 1998 JST, and (d) between 2000 on July 15 and 0600 on July 16 , 1998 JST.

isks, orange rectangles, and blue crosses, respectively. The observation locations of TEC are determined with the direction of the line-of-sight between satellites and receivers, and the assumed ionospheric height. If the assumed height is not proper, TECs are located at wrong positions. The validity of the assumed height is examined by the comparison between the TEC patterns derived from the different GPS satellites because the different GPS satellites have different line-ofsite directions. The ionospheric altitude is assumed at 300 $\mathrm{km}$ for the case of Fig. 6(b). The locations of the maxima and minima derived from the different satellites show good agreement. We examined the cases with $250 \mathrm{~km}, 300 \mathrm{~km}$, $350 \mathrm{~km}$, and $400 \mathrm{~km}$ ionospheric altitude, and found that the case with $300 \mathrm{~km}$ ionospheric altitude shows the best agreement. The perturbation components of TEC are used in this analysis. Thus, the ionospheric altitude estimated by this method is not the center altitude of the whole ionosphere, but the center altitude of the perturbation of the electron density. This analysis shows that the variations of the electron density inside TIDs occurred in the altitude range centered at $300 \mathrm{~km}$ altitude on this night. This altitude range was below the peak altitude of the $F$-region ionosphere and coincident with the altitude range where the 3 -m scale $F$-region FAIs were detected by the coherent scatter observation.

The altitude-time-intensity plot of the coherent echoes in other four nights, May 20, May 21, May 22, and July 15 in 1998, are plotted with the $F$-region peak altitude derived from the incoherent scatter observation of the MU radar in Fig. 7. The $F$-region FAIs appeared in three nights out of the four nights. The uplift of the ionosphere was detected in all of the time periods when the $F$-region FAIs were detected just as occurred in the event on May 18, 1998. The time periods when the $F$-region FAIs appeared were also coincident with the time periods when the amplitude of TIDs was large. In every case, the $F$-region FAIs occurred below the $F$-region peak height.

The dependency of occurrence of the $F$-region FAIs on the amplitude of TIDs was reported for the FRONT-1 campaign period by Saito et al. (2001). To see this relation in an extend time period, the data from 6 nights in June and July, 1997, nine nights in May, 1998 of the FRONT-1 campaign, and six nights of the FRONT-2 campaign in August, 1999 are studied. Standard deviations of TEC over Shigaraki within one-hour period are calculated to measure the 


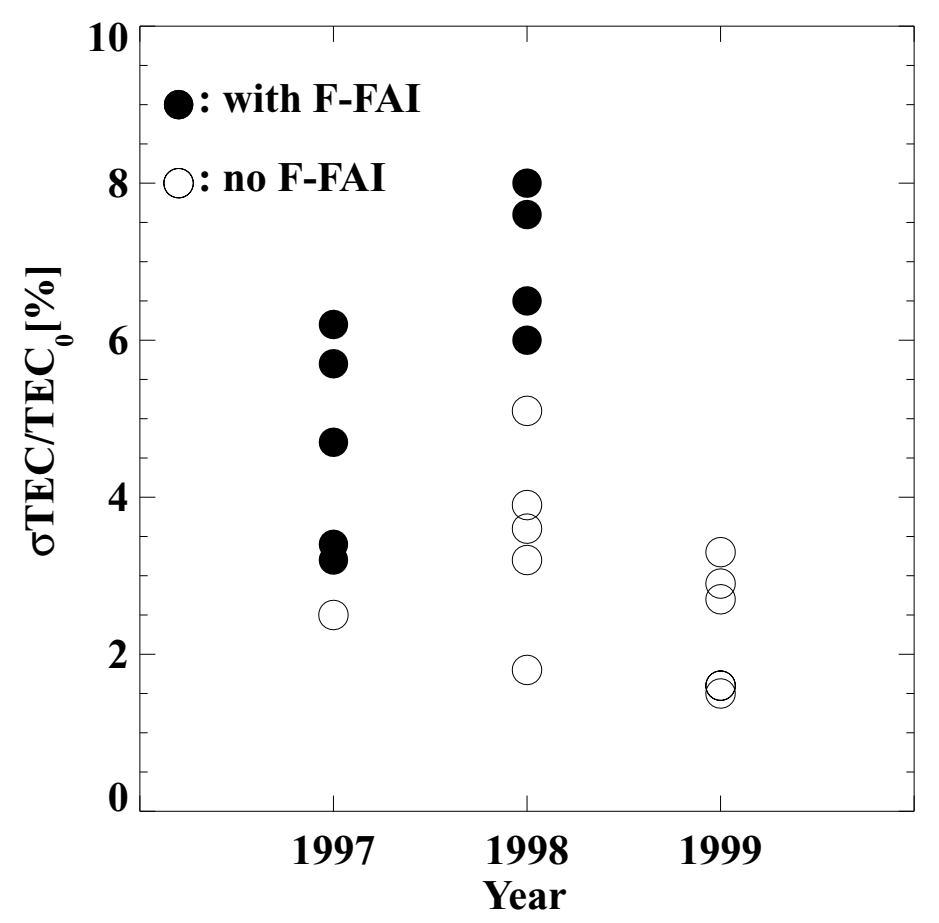

Fig. 8. Distribution of the ratios of the TEC standard deviation to the background value of TEC during the summer nighttime. Maximum values of the ratios for each nights are plotted. The ratios for the nights when the 3-m scale $F$-region field-aligned irregularities (FAIs) were detected by the MU radar are represented by solid circles. The ratios for the nights without the $F$-region FAIs are represented by open circles.

amplitude of TIDs. These standard deviations of TEC are good index of the medium scale TIDs (Saito et al., 2001). The ratios of the standard deviation of TEC to the background value of TEC are derived every hour. The maximum values of the ratio through one night are plotted in Fig. 8 for each day. The solid circles represent the maximum values for the nights when the $F$-region FAIs were detected by the coherent observation of the MU radar, and open circles represent the maximum values for the nights without the $F$ region FAIs. In 1999, two days had a same ratio, 1.6\%, and the circles are overlapped. The $F$-region FAIs have clear anti-correlation to the solar cycle (Fukao et al., 1991). They appeared at five nights out of six nights observation in 1997, and at four nights out of nine nights in 1998. No $F$-region FAIs were detected in the six nights in 1999. The amplitude of TIDs measured by the ratio of the perturbation component to the background value was lower in the high solar activity period, August in 1999 (SSN = 93.7) than in the low solar activity periods, June in 1997 (SSN = 12.7) and May in 1998 (SSN = 56.3). The nights when the $F$-region FAIs appeared had a tendency to have large ratio of the perturbation TEC to the background value. This tendency is clear in 1998.

\subsection{Horizontal distribution of the $F$-region FAIs and MSTIDs}

The multi-beam observation of the MU radar enables to detect the two-dimensional distribution of the $F$ region FAIs. The grayscales in Figs. 9 and 10 show the coherent echo from the 3-m scale $F$-region FAIs observed by the MU radar, and are over plotted by the color contour of the perturbation components of TEC detected by GEONET. The field-of-views of the MU radar coherent scatter observation are represented by solid line between $36^{\circ}$ and $40^{\circ}$ in latitude, and $132^{\circ}$ and $139^{\circ}$ in longitude. The observations of the coherent echoes are on a plane perpendicular to the geomagnetic field line. Thus, the nearest range of this field-of-view is about $150 \mathrm{~km}$ altitude and the furthest range of this fieldof-view is about $450 \mathrm{~km}$ altitude. Figure 9 shows the plots in every 10 minutes between 2339 and 0029 on June 12, 1997 JST. The $F$-region FAI formed band-like structures stretching from the northwest to the southeast as the wavefronts of the perturbation components of TEC. The alignment of these band-like structures and that of the TEC structures show excellent agreement. They traveled to the southwest in the same propagation velocity. The regions where the echoes from the $F$-region FAIs were detected correspond to the peak and the northeastern slope, where the gradient of the perturbation components of TEC points to the southwest. Figure 10 shows between 2140 and 2234 on July 3, 1997 JST in every 11 minutes. In this case the alignments of the $F$-region FAIs and the perturbation components of TEC were not clear as the case in Fig. 9. The $F$-region FAIs tended to occur at the peak and the northeastern slope in this night too.

\section{Discussion}

TIDs and 3-m scale FAIs were observed in the nighttime $F$-region ionosphere with the MU radar and GEONET in the FRONT campaigns of May 1998 and August 1999. The relations among the background electron density structures, the nighttime TIDs, and the $F$-region FAIs were revealed with these multi-instrument observations of the FRONT campaign.

The simultaneous observations of coherent scatter echoes 

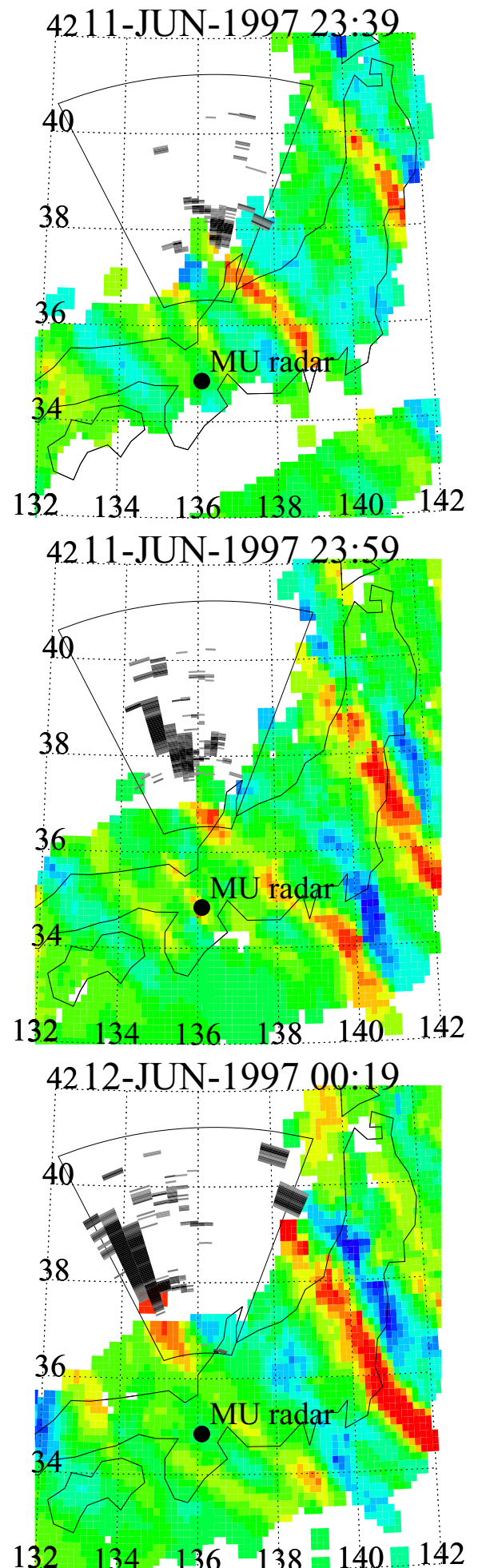
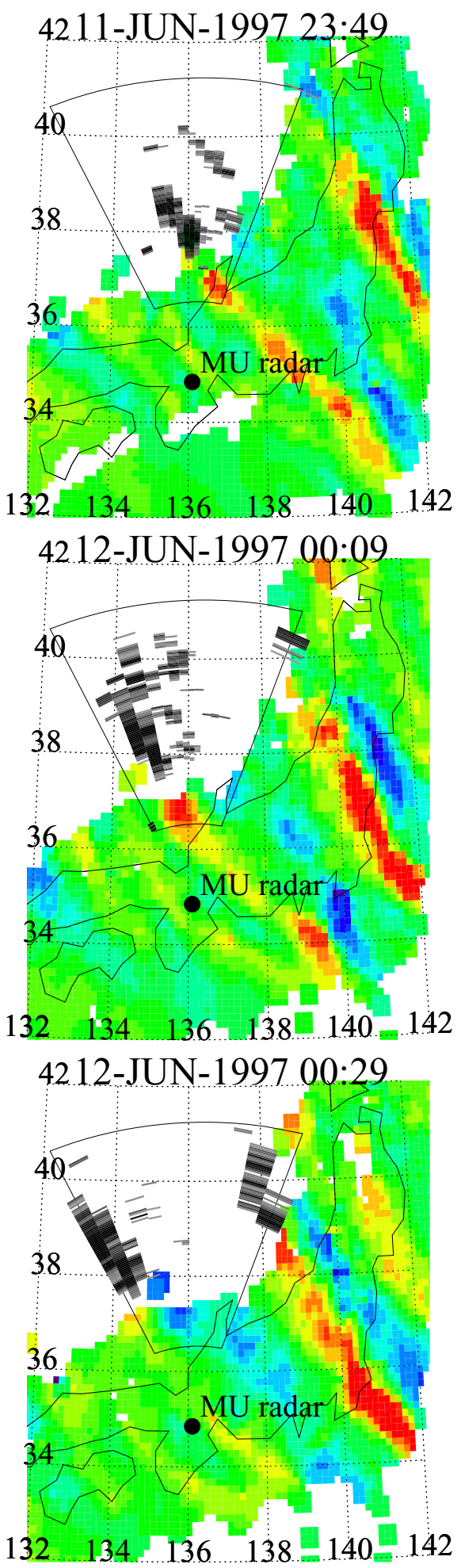

Fig. 9. Two-dimensional distribution of the perturbation component of total electron content detected by GEONET and the $F$-region FAIs detected by the MU radar in every 10 minutes between 2339 on June 11 and 0029 on June 12, 1997 JST. The amplitude of TEC is displayed by the color contour and the intensity of the coherent echoes from the $F$-region FAI is displayed by gray scale. The field of views of the MU radar coherent echo observation is represented by solid lines between $36^{\circ}$ and $40^{\circ}$ in latitude, and $132^{\circ}$ and $139^{\circ}$ in longitude.

and incoherent scatter echoes by the MU radar revealed that the 3-m scale irregularities occurs on the bottomside of the $F$-region ionosphere. The spectrum of the coherent echoes from the $F$-region FAIs is categorized to the type 2 . Thus, the $F$-region FAIs are believed to be generated by the gra- dient drift instability (Fukao et al., 1991; Kelley and Fukao, 1991). The gradient drift instability is excited when the electric current and the spatial gradient of the electric conductivity are intense enough. To see the average status of the conductivity distribution in the mid-latitude ionosphere, the 

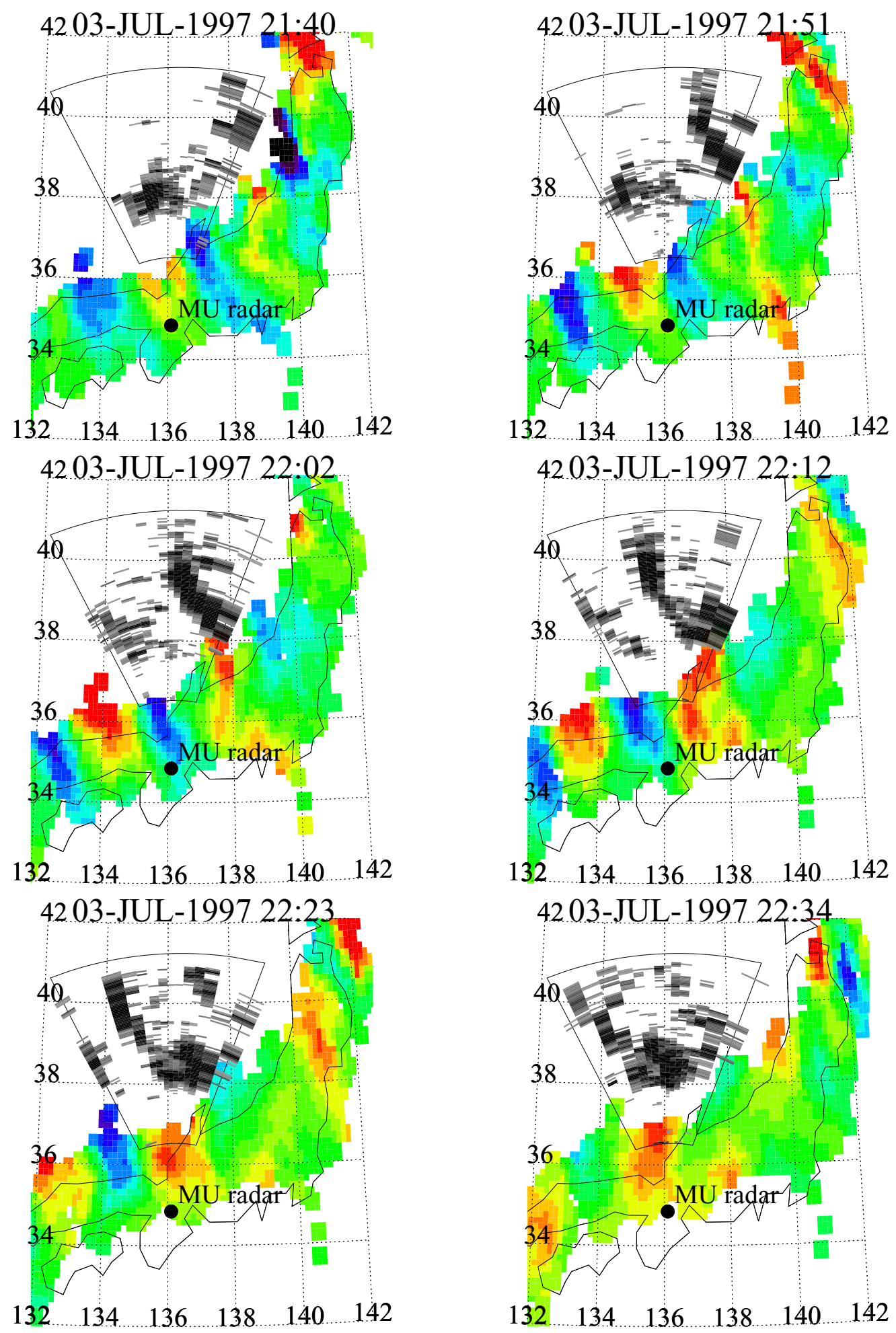

$\begin{array}{llllll}132 & 134 & 136 & 138 & 140 & 142\end{array}$

Fig. 10. Same as Fig. 9 for between 2140 and 2234 on July 3, 1997 JST in every 11 minutes.

ionospheric conductivity is calculated with the IRI model for the ionized atmosphere and the CIRA model for the neutral atmosphere (WDC for geomagnetism, Kyoto, 1999). The ratio of the vertical gradient of the Pedersen conductivity to the Pedersen conductivity derived by the model is plotted in the left side panel of Fig. 11. The conductivity at 0000 local time over Shigaraki was calculated for two months, July 1997 and July 1999. The inclination of the geomagnetic field line is $48.8^{\circ}$ at Shigaraki. Therefore, the perpendicular component of the vertical gradient of the conductivity contributes to the generation of the gradient drift instability. The Pedersen conductivity is proportional to the product of the electron density and the neutral density. The neutral density uniformly decreases as altitude increases. Thus, the peaks of the Pedersen conductivity are around $260 \mathrm{~km}$ altitude in the cases plotted in Fig. 11 and are well below the peaks of 


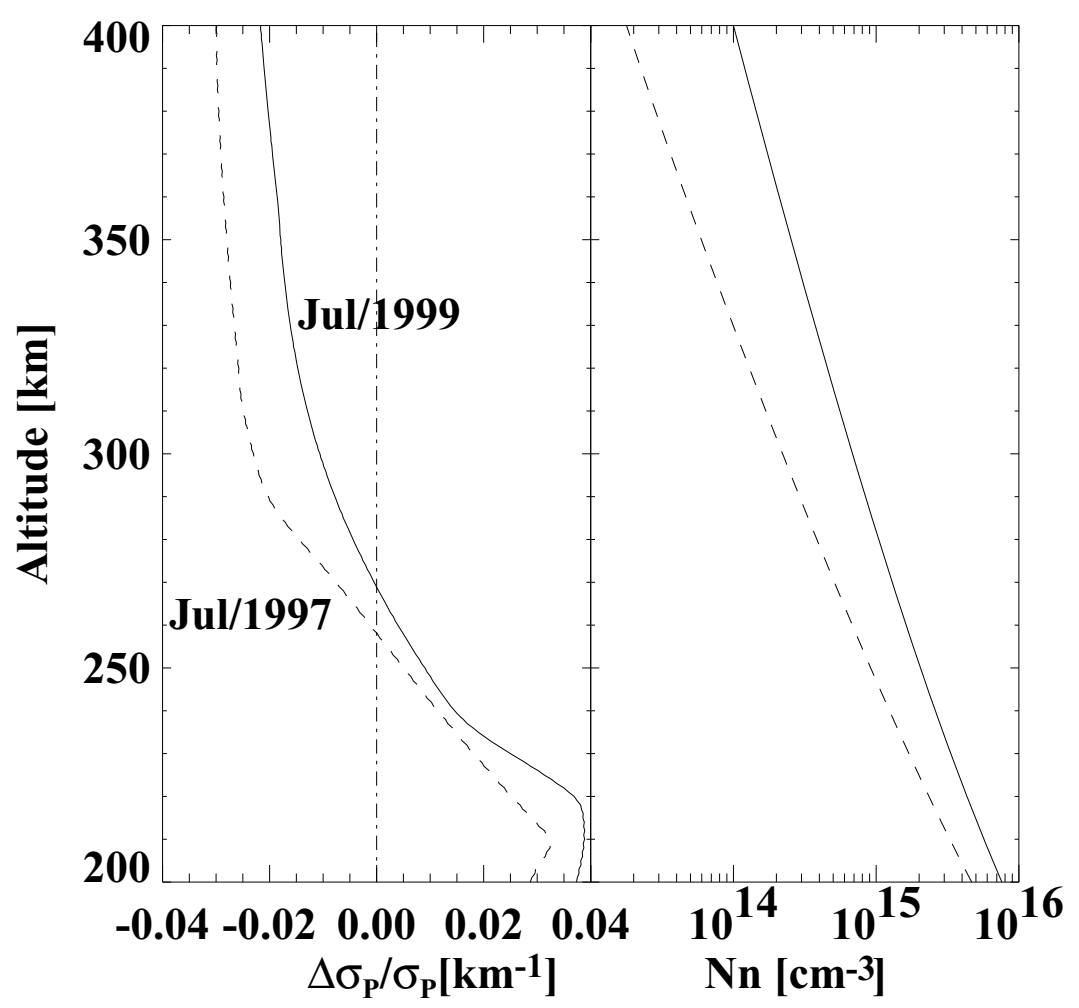

Fig. 11. Vertical gradient of the Pedersen conductivity (left-hand panel) and density of the neutral atmosphere (right-hand panel) that are calculated with IRI and CIRA models for 0000 local time over Shigaraki in July 1997 (dashed line) and July 1999 (solid line).

the electron density that are higher than $300 \mathrm{~km}$ altitude. In the altitude region where the $F$-region FAIs were detected by the MU radar, between $250 \mathrm{~km}$ and $350 \mathrm{~km}$ altitude, the vertical gradient of the Pedersen conductivity is downward. Therefore, the gradient perpendicular to the geomagnetic field line is southward and downward. In this altitude region, the conductivity gradient is larger in 1997 than that in 1999. The empirical model describes the average status of the ionosphere and its application for an individual day does not precisely describe the ionospheric condition of the individual day. However this solar cycle dependency of the conductivity gradient is reliable because it is mainly caused by the dependency of the scale height of the neutral atmosphere on the solar cycle. The right-hand side panel of Fig. 11 shows the density of the neutral atmosphere. The neutral atmosphere rapidly decreases with height in July 1997, when the sunspot number was 10.4. The scale height of the neutral atmosphere between $250 \mathrm{~km}$ and $350 \mathrm{~km}$ altitude is $37 \mathrm{~km}$. The scale height for this altitude range in July 1999 is $48 \mathrm{~km}$ by the model. The $F$-region FAIs have clear anti-correlation to the solar cycle as seen in Fig. 8 and reported by Fukao et al. (1991). At mid-latitudes, the electrical coupling between the topside and bottomside of the ionosphere by the geomagnetic field line is important and prevents the growth of the large scale ionospheric instability. However, the interchange instability proposed by Maruyama (1990) would generate the irregularities even when the electrical coupling between the topside and the bottomside is completely established. Furthermore the small scale electric field shortcircuits nearby the source region and does not completely map along the geomagnetic field line. Therefore we believe that the solar cycle dependency of the vertical gradient of the Pedersen conductivity on the bottomside of the $F$-region is one of the causes of the anti-correlation of the 3-m scale $F$ region FAIs to the solar activity. More precise observations of the vertical profile of ionospheric electron and the theoretical studies on the growth of the ionospheric irregularities in the mid-latitude ionosphere are necessary to evaluate this process.

The $F$-region FAIs had a tendency to appear in the time period when the modulations of TEC by TIDs were intense as seen in Fig. 8. Furthermore, some of FAIs formed similar wave front structures as those of TEC and traveled to the southwest in the similar velocity to that of TEC as seen in Fig. 9. Therefore, we suppose that the nighttime TIDs play an important role to generate the 3-m scale irregularities in the mid-latitude $F$-region ionosphere. The FAIs tend to appear at peak and the northeastern slope of TEC structures though at some times they appeared at any phases of TEC structures. In the altitude region where the FAIs are observed, the conductivity gradient caused by the vertical distribution of the atmosphere is downward and southward as seen in Fig. 11, and TIDs modulate the ionized atmosphere significantly in this altitude region. Therefore, the horizontal southwestern gradient generated by TIDs and the southward gradient by the vertical distribution of the conductivity on the bottomside of the $F$-region make the conductivity gradient intense at the northeastern slope of TIDs and 3-m scale irregularities are easily to be generated by the gradient drift instability. The good coincidence between the structures of 
the perturbation component of TEC and the $F$-region FAIs indicates that the gradient of the background TEC whose temporal scale size is larger than one hour is not large during these events. The $F$-region FAIs tend to occur in the northeastern slope of TIDs as shown in Figs. 9 and 10. If the $\mathrm{E} \times \mathrm{B}$ type instability causes the generation of the small scale irregularities, the electric current is expected to direct to northwest for those events.

When the electric field and/or the neutral wind are strong enough, the FAIs could occur not only in the region with strongest conductivity gradient but at any phases of TIDs as seen at 2234 on July 3, 1997, shown in Fig. 10. Though the data set is limited, the TEC observation of GEONET revealed that the amplitude of the nighttime TIDs is anticorrelation with the solar activity. This anti-correlation also causes the anti-correlation of the occurrence of the $F$-region FAIs. The physical mechanism of this anti-correlation of the TID amplitude has not been clarified. The Perkins instability is one of the explanations because the growth rate of the Perkins instability anti-correlates to the solar activity (Kelley and Fukao, 1991). The vertical structure of electron density in MSTIDs would provide the clue of their generation mechanism. If the polarization electric field is the primary driving force, as in the case of the Perkins instability, the structure is expected to be field-aligned (Perkins, 1973). If the neutral wind is the primary driving force, the electron density structure is not necessary to be field-aligned as discussed in Hines (1964). The temporal and spatial resolutions of vertical electron density profile observation by the MU radar were not high enough to discuss whether the structure is filed-aligned or not. The reconstruction of the vertical structure from GPS data derived by the ground-based receiver is not straightforward and beyond the scope of this paper.

TEC observation of the GPS network had frequently detected clearly structured wave fronts traveling to the southwest. These TEC structures had been attributed to the electron density variations in the $F$-region ionosphere because the largest part of total electron content between the ground level and the satellite orbit $(20,200 \mathrm{~km})$ is the content inside the $F$-region (Saito et al., 1998). The simultaneous observation of the electron density profile by the MU radar and the TEC by GEONET in the FRONT campaign directly clarified that the variations of TEC are attributed to the variations of electron density around and below the $F$-region peak. Saito et al. (2001) reported that the traveling structures of the 630 $\mathrm{nm}$ band airglow, which were detected with five all-sky cameras in the FRONT-1 campaign, showed an excellent coincidence with the TID structures of TEC. The plasma density variation on the bottomside of the $F$-region detected by the MU radar incoherent scatter observation is expected to generate the variations of $630 \mathrm{~nm}$ band airglow. The amplitude of the airglow variation was reported to be larger than that of TEC variations. The ratio of $630 \mathrm{~nm}$ airglow variations to the background value of airglow was $26 \%$, when the ratio of TEC variations to the background value was $8 \%$ on May 22, 1998. This tendency is consistent with the observation on August 08, 1999, which is presented in Fig. 4. The electron density variation between $327 \mathrm{~km}$ and $370 \mathrm{~km}$ altitudes, was $7.5 \%$ when the TEC variation was $2.5 \%$. The variations of electron content below the $F$-region peak are larger than that on the topside of the ionosphere, and cause both the variations in TEC and the large amplitude airglow variations. Ogawa et al. (2002) theoretically calculate the relation between the electron density variation and the 630 $\mathrm{nm}$ band airglow variations using SUPIM model.

The topside ionosphere was not to be significantly affected by TIDs. The result of the comparison of TEC structures derived with different GPS satellites, which is shown in Fig. 6, also confirms that the center of the variations is on the bottomside of the $F$-region even though the center of the whole TEC is at the $F$-region peak for the Chapman layer, the basic structure function for the whole ionosphere. We believe that the both of the neutral atmosphere and the ionized atmosphere contribute to this altitudinal distribution of the plasma density perturbations through the diffusion coefficient and the electric conductivity. Further theoretical studies including non-linear process with the both atmospheres are necessary to explain this phenomenon.

As seen Figs. 3, 5, and 7, intense TIDs and $F$-region FAIs accompanied the uplifts of the $F$-region ionosphere. The temporal scale of these uplifts was longer than that of TIDs. In the event of August 8, 1999, two intense TID structures were observed over Shigaraki during the $F$-region uplift, as seen in Fig. 3, and in the event of May 18, 1998, four intense TIDs were seen during the uplift as seen in Figs. 5 and 6. The Doppler velocity of coherent echo from the $F$-region FAIs indicates that this motion of the $F$-region would be driven by an electric field. This uplift seems to enhance the TID amplitude and trigger the $F$-region FAIs. The relations among this uplift, TID and FAIs have not been explained. Kelley et al. (2000) reported a reproducible signature of northward/upward electric field associated with the uplift of the $F$-region ionosphere, which was associated with $630 \mathrm{~nm}$ band airglow structures.

The incoherent scatter observations of the MU radar are severely contaminated by the echoes of sidelobes from the $E$-region FAIs. The contamination in the FRONT-1 campaign was so intense that electron density profiles on the bottomside of the ionosphere were often hard to identify. The contamination in the FRONT-2 campaign was less intense. This tendency of the contamination by the $E$-region FAIs anti-correlated to the amplitude of TIDs. The clearest incoherent scatter observation with least contaminations in the FRONT-2 campaign period is the observation in the night when the amplitude of TIDs was smallest in the period, August 08, 1999, as displayed in Figs. 3 and 4. This indicates that the activity of TIDs was correlated with the activity of the $E$-region FAIs, though the echoes from the radar sidelobes cannot precisely measure the activity of the $E$-region FAIs. Assuming that the gravity waves propagating from the lower atmosphere seed the TIDs in the $F$-region, the coincidence between the activities of the $E$-region FAIs and TIDs is reasonable. This relationship was specified by Bowman et al. (1994) using the MU radar observation and ionosonde observation. Further precise observations are necessary to clarify this relation.

\section{Summary}

The nighttime traveling ionospheric disturbances (TIDs) 
and the $F$-region 3-m scale field-aligned irregularities (FAIs) were simultaneously observed with the MU radar and GEONET, a GPS network, in Japan during the FRONT campaign periods in May 1998 and August 1999. TIDs were detected by the total electron content (TEC) observations of GEONET in 21 nights out of the 24 nights of observations during the campaign periods. The incoherent scatter observations of the MU radar revealed that the electron density variations that were attributed to the TEC variations of TIDs occurred around and below the peak of the $F$-region ionosphere, and the electron density variations on the topside of the ionosphere were less intense than that on the bottomside. The TEC observations with multiple-satellites confirmed that the center of the electron density variations of TIDs was $300 \mathrm{~km}$ altitude. The amplitudes of TIDs showed a tendency to be enhanced when the ionosphere was uplifted for a few hours. The ratio of the amplitude of TIDs to the background value of TEC tended to be high in the low solar activity periods.

The 3-m scale field-aligned irregularities were observed on the bottomside of the $F$-region when the amplitude of TIDs was large. The FAIs had a tendency to appear at the peak and on the northeastern slope of TIDs. This tendency indicates that the horizontal gradient of the electric conductivity generated by TIDs and the vertical gradient of the conductivity on the bottomside of the $F$-region would cause the $3-\mathrm{m}$ scale irregularities through the gradient drift instability. The occurrence rate of the $F$-region FAIs anti-correlated to the solar activity. This would be caused by the solar cycle dependences of the TID amplitudes and the vertical gradient of the Pedersen conductivity.

This phenomenon is characterized by various scale sizes, as the large-scale ionospheric dynamics including uplift of the ionosphere, the mesoscale disturbances referred to as TIDs, and the small-scale irregularities, $F$-region FAIs. Although the relations between them are being addressed by multi-instrument observations, further theoretical studies are necessary to understand its physical mechanism.

Acknowledgments. The MU radar belongs to and is operated by the Radio Science Center for Space and Atmosphere of Kyoto University. We acknowledge the Geographical Survey Institute, Japan for providing GEONET data used in this study. The ionosonde data of Kokubunji was provided by the Communication Research Laboratory, Japan. AS's research at Cornell University is supported by JSPS Postdoctal Fellowships for Research Abroad.

\section{References}

Bowman, G. G., S. Fukao, M. Yamamoto, and K. Igarashi, MU-radar recorded field-aligned irregularities in the F2 region and associated sporadic E disturbances, J. Geomag. Geoelectr., 46, 873-889, 1994.

Francis, S. H., A theory of medium-scale traveling ionospheric disturbances, J. Geophys. Res., 79, 5245-5260, 1974.

Fukao, S., J. P. McClure, A. Ito, T. Sato, I. Kimura, T. Tsuda, and S. Kato, First VHF radar observation of midlatitude F-region Field-Aligned Irregularities, Geophys. Res. Lett., 15, 768-771, 1988.

Fukao, S., M. C. Kelley, T. Shirakawa, T. Takami, M. Yamamoto, T. Tsuda, and S. Kato, Turbulent upwelling of the mid-latitude ionosphere 1. observational results by the MU radar, J. Geophys. Res., 96, 37253746, 1991.

Fukao, S., M. Yamamoto, R. T. Tsunoda, H. Hayakawa, and T. Mukai, The SEEK (Sporadic-E Experiment over Kyushu) Campaign, Geophys. Res.
Lett., 25, 1761-1764, 1998.

Garcia, F. J., M. C. Kelley, J. J. Makela, and C.-S. Huang, Airglow observations of mesoscale low-velocity traveling ionospheric disturbances at mid-latitudes, J. Geophys. Res., 105, 18,407-18,416, 2000.

Hines, C. O., Internal Atomspheric Gravity Waves at Ionospheric Heights, Canadian J. Phys., 38, 1441-1481, 1964.

Jacobson, A. R., R. C. Carlos, R. S. Massey, and G. Wu, Observations of traveling ionospheric disturbances with a satellite-beacon radio interferometer: Seasonal and local time behavior, J. Geophys. Res., 100, 1653-1665, 1995.

Kelley, M. C. and S. Fukao, Turbulent upwelling of the mid-latitude ionosphere, 2, Theoretical framework, J. Geophys. Res., 96, 3747-3753, 1991.

Kelley, M. C. and C. A. Miller, Electrodynamics of midlatitude spread $F$ 3. Electrohydrodynamic waves? A new look at the role of electric field in thermospheric wave dynamics, J. Geophys. Res., 102, 11,539-11,547, 1997.

Kelley, M. C., J. J. Makela, A. Saito, N. Aponte, M. Sulzer, and S. A. Gonzalez, On the electrical structure of airglow depletion/height layer bands over Arecibo, Geophys. Res. Lett., 27, 2837-2840, 2000.

Kubota, M., K. Shiokawa, M. K. Ejiri, T. Ogawa, T. Sakanoi, H. Fukunishi, M. Yamamoto, S. Fukao, A. Saito, and S. Miyazaki, Tracking of wavelike structures in the OI 630-nm nightglow over Japan using an allsky imagers network during FRONT campaign, Geophys. Res. Lett., 27 4037-4040, 2000 .

Maruyama, T., $\mathrm{E} \times \mathrm{B}$ Instability in the $F$ region at low- to midlatitude, Planet. Space Sci., 38, 273-285, 1990.

Mendillo, M., J. Bauumgardner, D. Nottingham, J. Aarons, B. Reinisch, J. Scali, and M. C. Kelley, Investigations of thermospheric-ionospheric dynamics with 6300 - $\AA$ images from the Arecibo observatory, J. Geophys. Res., 102, 7331-7343, 1997

Miller, C. A., W. E. Swartz, M. C. Kelley, M. Mendillo, D. Nottingham, J. Scali, and B. Reinisch, Electrodynamics of midlatitude spread $F$ 1. Observations of unstable, gravity wave-induced ionospheric electric fields at tropical latitudes, J. Geophys. Res., 102, 11,521-11,532, 1997.

Ogawa, T., N. Balan, Y. Otsuka, K. Shiokawa, C. Ihara, T. Shimomai, and A. Saito, Observations and modeling of $630 \mathrm{~nm}$ airglow and total electron content associated with traveling ionospheric disturbances over Shigaraki, Japan, Earth Planets Space, 54, this issue, 45-56, 2002.

Otsuka, Y., T. Ogawa, A. Saito, T. Tsugawa, S. Fukao, and S. Miyazaki, A new technique for mapping of total electron content using GPS network in Japan, Earth Planets Space, 54, this issue, 63-70, 2002.

Perkins, F., Spread F and ionospheric currents, J. Geophys. Res., 78, 218 226, 1973.

Saito, A., S. Fukao, and S. Miyazaki, High resolution mapping of TEC perturbations with the GSI GPS network over Japan, Geophys. Res. Lett., 25, 3079-3082, 1998

Saito, A., M. Nishimura, M. Yamamoto, M. Kubota, K. Shiokawa, Y. Otsuka, T. Tsugawa, S. Fukao, T. Ogawa, M. Ishii, T. Sakanoi, and S Miyazaki, Traveling ionospheric disturbances detected in the FRONT campaign, Geophys. Res. Lett., 28, 689-692, 2001.

Shiokawa, K., M. K. Ejiri, Y. Otsuka, T. Ogawa, M. Kubota, K. Igarashi, A. Saito, and T. Nakamura, Multi-point observation of short-period mesospheric gravity waves over Japan during the FRONT campaign, Geophys. Res. Lett. 27, 4057-4060, 2000.

Shiokawa, K., Y. Otsuka, M. K. Ejiri, Y. Sahai, T. Kadota, C. Ihara, T. Ogawa, K. Igarashi, S. Miyazaki, and A. Saito, Imaging observations of the equatorward limit of mid-latitude traveling ionospheric disturbances, Earth Planets Space, 54, this issue, 57-62, 2002.

Swartz, W. E., M. C. Kelley, J. J. Makela, S. C. Collins, E. Kudeki, S. Franke, J. Urbina, N. Aponte, M. P. Sulzer, and S. A. Gonzalez, Coherent and incoherent scatter radar observations during intense mid-latitude spread F, Geophys. Res. Lett., 27, 2829-2832, 2000.

Taylor, M. J., J.-M. Jahn, S. Fukao, and A. Saito, Possible evidence of gravity wave coupling into the midlatitude $\mathrm{F}$ region ionosphere during the SEEK campaign, Geophys. Res. Lett., 25, 1801-1804, 1998.

WDC for geomagnetism, Kyoto, Ionospheric conductivity model, http: //swdcdb.kugi.kyoto-u.ac.jp/sigcal/, 1999.

A. Saito (e-mail: saito@arc.ee.cornell.edu), M. Nishimura, M Yamamoto, S. Fukao, T. Tsugawa, Y. Otsuka, S. Miyazaki, and M. C. Kelley 